\title{
VOID HIERARCHY IN THE NORTHERN LOCAL VOID
}

\author{
U. LINDNER AND K.J. FRICKE \\ Universitäts-Sternwarte, Göttingen, Germany \\ AND
}

J. EINASTO AND M. EINASTO

Tartu Astrophysical Observatory, Tóravere, Estonia

\section{Introduction}

Empirical studies of the Large-Scale Structure in the nearby Universe come in two complementary modes, namely the investigation of either the distribution of luminous matter or voids: (i) The description of the galaxy and cluster distribution employs correlation functions, clustering analysis, topological methods, et cetera. (ii) The investigation of the empty regions between systems of galaxies uses void probability functions, mean diameters of voids, the compilation of void catalogues, and so forth.

Here we focus on the study of voids and for this purpose it must be clarified what voids are. Two essentially different definitions are in use: Voids can be defined as (i) regions of low density in a suitably smoothed density field of the galaxy distribution or as (ii) regions completely empty of a certain type of object, e.g. rich or poor galaxy clusters or galaxies of a specified morphological type or luminosity limit.

Figure $1 \mathrm{a}$ ) shows the distribution of rich galaxy clusters from the AbellACO catalogue which constitute the cores of superclusters. The large voids between superclusters are called supervoids. They are by definiton devoid of rich clusters and have diameters of about $100 h^{-1} \mathrm{Mpc}$. They are the largest known voids in the Universe. Superclusters are connected and form a chess board like regular network.

Figure $1 \mathrm{~b}$ ) gives a closer look to the Northern Local Void (NLV) region indicated in Figure 1a). This closest supervoid which is bordered by the Hercules-, Coma- and Local Supercluster is not completely empty but contains systems of galaxies of various richness. This finding motivates a 

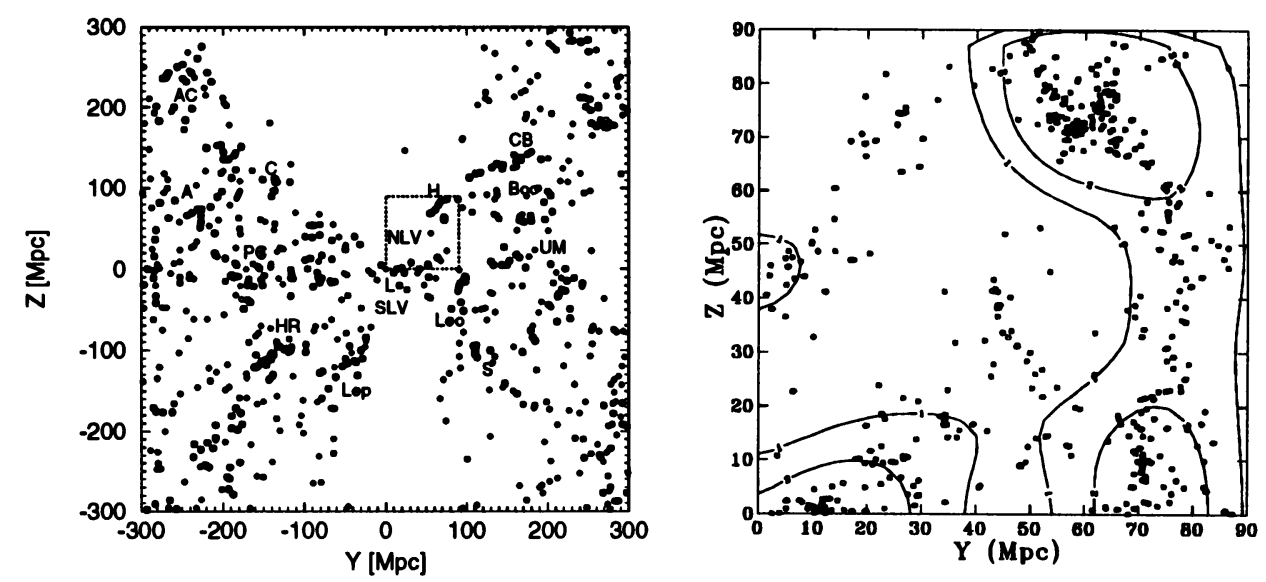

Figure 1. a) rich (Abell-ACO) clusters in supergalactic coordinates $\mathrm{X}, \mathrm{Y}, \mathrm{Z}$; sheet is $\Delta \mathrm{X}=200 h^{-1} \mathrm{Mpc}$ thick (M. Einasto 1995); b) galaxies in the NLV region (indicated by the square in Figure 1 a)

more detailed study of the Large-Scale structures in this low density region concerning morphological type and luminosity of galaxies.

\section{Basic Data}

The region of the Northern Local Void (NLV) covers $12^{h}<\alpha<18^{h}, \delta>0^{\circ}$ on the sky and is about $15000 \mathrm{~km} \mathrm{~s}^{-1}$ deep in redshift space. Using a Hubble constant of $\mathrm{H}=h 100 \mathrm{~km} \mathrm{~s}^{-1} \mathrm{Mpc}^{-1}$ this corresponds to a distance of 150 $h^{-1} \mathrm{Mpc}$.

Redshifts for poor clusters of galaxies from the Zwicky et al. (196168) catalogue have been estimated by E. Tago (1993). Galaxy redshifts are taken from the ZCAT (Huchra, 1994) and various other sources, mainly the Arecibo $21 \mathrm{~cm}$ survey (Giovanelli et al., 1992). From this redshift compilation which is complete up to $m_{\text {lim }}=15.5$ we selected three absolute magnitude limited cubic samples of different size $\left(\mathrm{L}=60,90\right.$ and $\left.120 h^{-1} \mathrm{Mpc}\right)$ for galaxies of early type ( $\mathrm{E}$ and S0) and all morphological types. Using the completeness limit $m_{\text {lim }}=15.5$ the luminosity limits of the samples are $M_{\text {lim }}=-18.8,-19.7$ and -20.3 , respectively.

In Figure 2 we present cross sections through cubic samples $(L=$ $60 h^{-1} \mathrm{Mpc}$ ) for galaxies of all types with different luminosity limit (as indicated in the legend). Bright galaxies are situated in the centers of galaxy systems. Fainter galaxies gather around these centers and in some case they form new subtle structures (filaments or sheets) between these systems.

For detailed quantitative studies we chose a void analysis applying the empty sphere method which is particularly sensitive to subtle structures in 


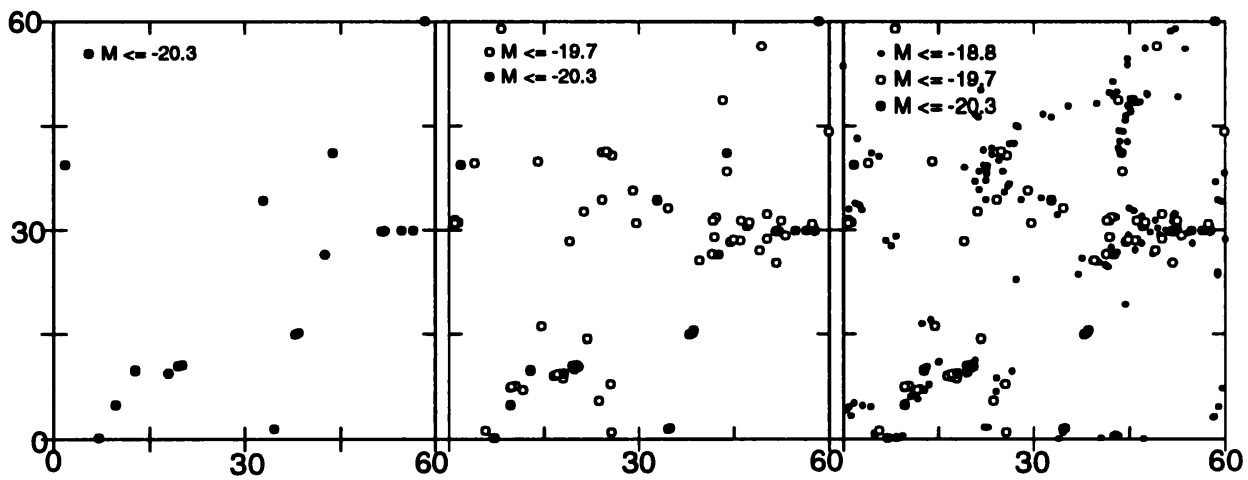

Figure 2. Cross sections through cubic samples of galaxies with different luminosity limit (cf. legend) in the NLV region. The cube size is $L=60 h^{-1} \mathrm{Mpc}$.

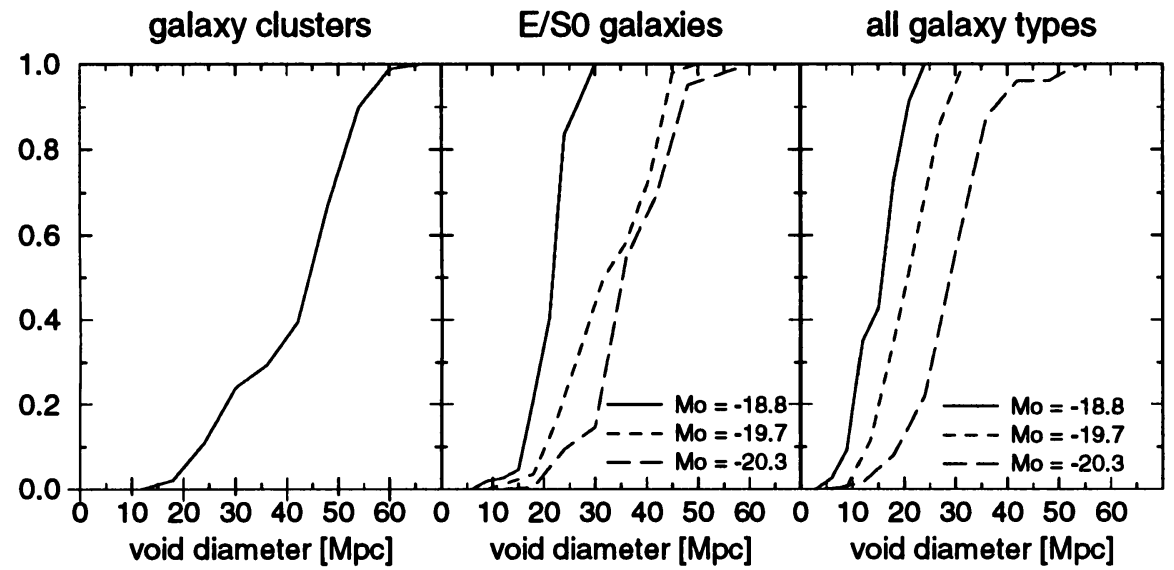

Figure 3. Cumulative distributions of void diameters

the galaxy distribution. For a more detailed description of the methods we refer the reader to Lindner et al. (1995).

\section{Statistics of void diameters}

In Figure 3 we present cumulative distributions of void diameters for Zwicky clusters, early type galaxies and galaxies of all morphological type. Three different luminosity limited galaxy samples are indicated. We see that voids surrounded by early type galaxies are larger than those found in respective samples of all morphological types and fainter galaxies outline smaller voids than bright galaxies. The distribution of void diameters in the case of clusters is similar to that of bright elliptical galaxies resembling the fact 
that these galaxies reside predominantly in cluster cores. This dependence of void diameters on the type of object and the galaxy luminosity is quantified by the mean values given in Table 1 with random errors $\epsilon$ and the rms scatter $\sigma$.

TABLE 1. Mean diameters of voids $\langle D\rangle$ surrounded by different objects

\begin{tabular}{rlr}
\hline \multicolumn{1}{c}{ type of object } & \multicolumn{1}{c}{$\langle D\rangle \pm \epsilon$} & $\sigma$ \\
\hline rich clusters (Abell/ACO) & $\approx 100 h^{-1} \mathrm{Mpc}$ \\
poor clusters (Zwicky) & $37.0 \pm 3.5 h^{-1} \mathrm{Mpc}$ & $11.7 h^{-1} \mathrm{Mpc}$ \\
elliptical galaxies $(M \leq-20.3)$ & $36.4 \pm 3.4 h^{-1} \mathrm{Mpc}$ & $9.2 h^{-1} \mathrm{Mpc}$ \\
galaxies brighter than $M_{0}=-20.3$ & $25.7 \pm 1.3 h^{-1} \mathrm{Mpc}$ & $6.4 h^{-1} \mathrm{Mpc}$ \\
galaxies brighter than $M_{0}=-19.7$ & $18.8 \pm 0.9 h^{-1} \mathrm{Mpc}$ & $5.3 h^{-1} \mathrm{Mpc}$ \\
galaxies brighter than $M_{0}=-18.8$ & $14.0 \pm 0.9 h^{-1} \mathrm{Mpc}$ & $4.0 h^{-1} \mathrm{Mpc}$ \\
\hline
\end{tabular}

The number of objects in various samples is very different, ranging from 1850 faint galaxies to 140 bright elliptical galaxies. Hence the question arises whether our results reflect a real property of the galaxy distribution or if they are caused only by a different number of objects. A comparison study to Poisson samples reveals statistic significance of our results (cf. Lindner et al. 1995).

\section{Void catalogues}

Void catalogues have been compiled by various authors: Kauffmann \& Fairall (1991) found cubic empty regions; Slezak, de Lapparent \& Bijaoui (1993) used a wavelet method to detect low density regions and El-Ad, Piran \& da Costa (1996) presented a new void search algorithm for automated void detection classifying galaxies into wall and field galaxies, the latter being allowed to lie inside voids. But all these investigators did not consider the different luminosity of galaxies.

We employed the empty sphere method to compile void catalogues for three different absolute magnitude limited galaxy samples. They are named A, B and C for $M_{l i m}=-20.3,-19.7$ and -18.8 , respectively, and contain the position and distance of void centers and the void diameter.

About one third of the voids in catalogue $A$ can be identified also in catalogue $B$ and a similar result is found for catalogues $B$ and $C$. The study of overlappings between voids from different catalogues shows that (i) systems of faint galaxies enter into voids outlined by bright galaxies and make their diameter smaller or that (ii) one or more faint galaxy systems split large voids into several subvoids. 


\section{Void hierarchy}

Our statistical studies reveal that void diameters depend on the type of object surrounding voids. Cluster defined voids are the largest voids and voids surrounded by early type galaxies are larger than those formed by galaxies of all morphological type. Furthermore we found that voids are smaller in samples of fainter galaxies.

Intersections between voids from different catalogues show that voids outlined by bright galaxies contain fainter galaxies and may be interlaced by systems of fainter galaxies which divide them into smaller subvoids.

These results indicate that voids form a hierarchical system resembling the hierarchy of galaxies, clusters of galaxies and superclusters.

TABLE 2. The hierarchy of voids

voids surrounded by rich clusters: supervoids
voids surrounded by poor (Zwicky) clusters of galaxies
voids surrounded by bright elliptical galaxies
voids surrounded by galaxies brighter than $M_{0}=-20.3$
voids surrounded by galaxies brighter than $M_{0}=-19.7$
voids surrounded by galaxies brighter than $M_{0}=-18.8$
voids surrounded by galaxies fainter than $M_{0}=-18.8$ (dwarf galaxies)?

As far as we know the upper limit of this hierarchy is given by the supercluster network with supervoids of about $100 h^{-1} \mathrm{Mpc}$ in diameter. By now the lower limit is not well determined. In our investigation the smallest voids are defined by galaxies from complete samples with absolute magnitude limit $M_{l i m}=-18.8$. Studies of incomplete samples fainter than this limit give strong hints that the void hierarchy may continue to dwarf galaxies. That means dwarf galaxies are not distributed homogeneously but follow the structures delineated by bright galaxies or form subtle filamentary substructures between dense galaxy systems (cf. Lindner et al. 1996).

\section{Conclusion}

Numerical simulations of voids by Regös \& Geller (1991), van de Weygaert \& van Kampen (1993), Dubinski et al. (1993), Sahni et al. (1994) agree with our picture of the present internal structure of supervoids found in the galaxy distribution of the NLV. They studied the evolution of structures inside voids and found that small (primordial) voids merge in course of time to form larger voids inside supervoids at later epochs, i.e. there was more substructure in the beginning than today. The existence of hierarchical 
substructures in supervoids rules out the non hierarchical hot dark matter model. The existence of structure at small and large scales favors a double power law power spectrum for primordial density fluctuations, as suggested by Einasto et al. or some mixed dark matter model.

Other, more distant supervoids may have similar internal structure. For example Szomoru et al. $(1993,1996)$ have found some galaxies in the Boötes Void which may be part of a similar web as we have found in the NLV. In general selection effects hinder to detect the full extend of faint structures. Deeper investigations like the ESO slice project (Vettolani et al. 1996) and Las Campanas Redshift Survey (Shectman et al. 1996) confirm the existence of a supercluster - supervoid network and faint structures inside the supervoids. The next generation redshift surveys may help to reduce the problems connected with selection effects and to establish the existence and lower limit of the void hierarchy.

\section{References}

Dubinski, J., da Costa, L.N., Goldwirth, D.S., Lecar, M., Piran, T., 1993, ApJ 410, 458

Einasto, M., 1995, in: Large Scale Structure in the Universe, 11th Potsdam international workshop, World Scientific, 1995

El-Ad, H., Piran, T., da Costa, L.N., 1996, ApJL 462, L13

Giovanelli, R., Haynes, M., Freudling, W., 1992, private communication

Huchra, J.P. 1994, ZCAT (compilation of galaxy redshifts and manual available via $\mathrm{ft}_{\mathrm{p}}$ )

Kauffmann, G., Fairall, A.P., 1991, MNRAS 248, 313

Lindner, U., Einasto, J., Einasto, M., Frendling, W., Fricke, K.J., and Tago, E., 1995, A\&A 301, 329

Lindner, U., Einasto, M., Einasto, J., Freudling, W., Fricke, K.J., Lipovetsky, V., Pustilnik, S., Izotov, Y., and Richter, G., 1996, A\&A 314, 1

Regös, E., Geller, M.J., 1991, ApJ 377, 14

Sahni, V., Sathyaprakash, B.S., Shandarin, S.F., 1994, ApJ 431, 20

Shectman, S.A., Landy, S.D., Oemler, A., Tucker, D.L., Lin, H., Kirshner, R.P., Schechter, P.L., (1996), ApJ 470, 172

Slezak, E., de Lapparent, V., Bijaoui, A., 1993, ApJ 409, 517

Szomoru, A., van Gorkom, J.H., Gregg, M., de Jong, R.S., 1993, AJ 105, 464

Szomoru, A., van Gorkom, J.H., Gregg, M., 1996, AJ 111, 2141

Tago, E., 1993, redshift compilation of NEARBY Zwicky clusters, private communication van de Weygaert, R., van Kampen, E., 1993, MNRAS 263, 481

Vettolani et al. 1996

Zwicky,F., Wield, P., Herzog, E., Karpowicz M., Kowal, C.T., 1961-68. Catalogue of Galaxies and of Clusters of Galaxies, 6 volumes. Pasadena, California Inst. Tech. 